\title{
Rational quartic reciprocity
}

by

\section{FrAnz LEMMERMEYER (Heidelberg)}

In 1985, K. S. Williams, K. Hardy and C. Friesen [11] published a reciprocity formula that comprised all known rational quartic reciprocity laws. Their proof consisted in a long and complicated manipulation of Jacobi symbols and was subsequently simplified (and generalized) by R. Evans [3]. In this note we give a proof of their reciprocity law which is not only considerably shorter but also sheds some light on the raison d'être of rational quartic reciprocity laws. For a survey on rational reciprocity laws, see E. Lehmer [7].

We want to prove the following

Theorem. Let $m \equiv 1 \bmod 4$ be a prime, and let $A, B, C$ be integers such that

$$
\begin{gathered}
A^{2}=m\left(B^{2}+C^{2}\right), \quad 2 \mid B, \\
(A, B)=(B, C)=(C, A)=1, \quad A+B \equiv 1 \bmod 4 .
\end{gathered}
$$

Then, for every odd prime $p>0$ such that $(m / p)=+1$,

$$
\left(\frac{A+B \sqrt{m}}{p}\right)=\left(\frac{p}{m}\right)_{4} .
$$

Proof. Let $k=\mathbb{Q}(\sqrt{m})$; then $K=\mathbb{Q}(\sqrt{m}, \sqrt{A+B \sqrt{m}})$ is a quartic cyclic extension of $\mathbb{Q}$ containing $k$, as can be verified quickly by noting that $A^{2}-m B^{2}=m C^{2}=(\sqrt{m} C)^{2}$ and $\sqrt{m} C \in k \backslash \mathbb{Q}$. We claim that $K$ is the quartic subfield of $\mathbb{Q}\left(\zeta_{m}\right)$, the field of $m$ th roots of unity. This will follow from the theorem of Kronecker and Weber once we have seen that no prime $\neq m$ is ramified in $K / \mathbb{Q}$. But the identity

$$
2(A+B \sqrt{m})(A+C \sqrt{m})=(A+B \sqrt{m}+C \sqrt{m})^{2}
$$

shows $K=k(\sqrt{2(A+C \sqrt{m})})$, and so the only odd primes that are possibly ramified in $K / k$ are common divisors of $A^{2}-m B^{2}=m C^{2}$ and $A^{2}-m C^{2}$ $=m B^{2}$. Since $B$ and $C$ are assumed to be prime to each other, only 2 and $m$ can ramify. Now $\sqrt{m} \equiv 1 \bmod 2($ since $m \equiv 1 \bmod 4)$ implies $B \sqrt{m} \equiv$ $B \bmod 4$, and we see $A+B \sqrt{m} \equiv A+B \equiv 1 \bmod 4$, which shows that 2 is not ramified in $K / k$ (and therefore not ramified in $K / \mathbb{Q}$ ). 
The reciprocity formula will follow by comparing the decomposition laws in $K / \mathbb{Q}$ and $\mathbb{Q}\left(\zeta_{m}\right) / \mathbb{Q}$ : if $(m / p)=+1$, then $p$ splits in $k / \mathbb{Q}$; if $f>0$ is the smallest natural number such that $p^{f} \equiv 1 \bmod m$ (here we have to assume $p>0)$, then $p$ splits into exactly $g=(m-1) / f$ prime ideals in $\mathbb{Q}\left(\zeta_{m}\right)$, and

$$
\begin{aligned}
\left(\frac{p}{m}\right)_{4}=1 & \Leftrightarrow p^{(m-1) / 4} \equiv 1 \bmod m \Leftrightarrow f \text { divides } \frac{1}{4}(m-1)=\frac{1}{4} f g \\
\Leftrightarrow & g \equiv 0 \bmod 4 \\
\Leftrightarrow & \text { the degree of the decomposition field } Z \text { of } p \\
& \text { is divisible by } 4 \\
& \left.\Leftrightarrow Z \text { contains } K \text { (because } \operatorname{Gal}\left(\mathbb{Q}\left(\zeta_{m}\right) / \mathbb{Q}\right) \text { is cyclic }\right) \\
& \Leftrightarrow p \text { splits completely in } K / \mathbb{Q} \\
& \Leftrightarrow\left(\frac{A+B \sqrt{m}}{p}\right)=1 .
\end{aligned}
$$

This completes the proof of the theorem.

Letting $m=2$ and replacing the quartic subfield of $\mathbb{Q}\left(\zeta_{m}\right)$ used above by the cyclic extension $\mathbb{Q}(\sqrt{2+\sqrt{2}})$ contained in $\mathbb{Q}\left(\zeta_{16}\right)$ yields the equivalence (3) $\left(\frac{A+B \sqrt{2}}{p}\right)=1 \Leftrightarrow p$ splits in $\mathbb{Q}(\sqrt{2+\sqrt{2}}) \Leftrightarrow p \equiv \pm 1 \bmod 16$, stated in a slightly different way in [11].

Formula (1) differs from the one given in [11], which reads

$$
\left(\frac{A+B \sqrt{m}}{p}\right)=(-1)^{(p-1)(m-1) / 8}\left(\frac{2}{p}\right)\left(\frac{p}{m}\right)_{4},
$$

where $A, B, C>0, B$ is odd and $C$ is even. Formula (2) shows that

$$
\left(\frac{A+B \sqrt{m}}{p}\right)=\left(\frac{2}{p}\right)\left(\frac{A+C \sqrt{m}}{p}\right)
$$

and so, for $B$ even and $C$ odd, (4) is equivalent to

$$
\left(\frac{A+B \sqrt{m}}{p}\right)=(-1)^{(p-1)(m-1) / 8}\left(\frac{p}{m}\right)_{4} .
$$

Now $A \equiv 1 \bmod 4$ since $A^{2}=m\left(B^{2}+C^{2}\right)$ is the product of $m \equiv$ $1 \bmod 4$ and of a sum of two relatively prime squares, and we have $A+B \equiv$ $1 \bmod 4 \Leftrightarrow 4 \mid B \Leftrightarrow m \equiv 1 \bmod 8$. The sign of $B$ is irrelevant, therefore

$$
\left(\frac{-1}{p}\right)^{B / 2}=(-1)^{(p-1)(m-1) / 8} .
$$

This finally shows that (1) is in fact equivalent to (4). 
Another version of (1) which follows directly from (5) is

$$
\left(\frac{A+B \sqrt{m}}{p}\right)=\left(\frac{p^{*}}{m}\right)_{4}
$$

where $A, B>0$ and $p^{*}=(-1)^{(p-1) / 2} p$.

Formula (1) can be extended to composite values of $m$ (where the prime factors of $m$ satisfy certain conditions given in [11] ) in very much the same way as Jacobi extended the quadratic reciprocity law of Gauss; this extension, however, is not needed in deriving the known rational quartic reciprocity laws of K. Burde [1], E. Lehmer [6, 7] and A. Scholz [9]. These follow from (1) by assigning special values to $A$ and $B$, in other words: they all stem from the observation that the quartic subfield $K$ of $\mathbb{Q}\left(\zeta_{m}\right)$ can be generated by different square roots over $k=\mathbb{Q}(\sqrt{m})$.

The fact that (1) is valid for primes $p \mid A B C$ (which has not been proved in [11]) shows that we no longer have to exclude the primes $q \mid a b$ in Lehmer's criterion (as was necessary in [11]), and it allows us to derive Burde's reciprocity law in a more direct way: let $p$ and $q$ be primes $\equiv 1 \bmod 4$ such that $p=a^{2}+b^{2}, q=c^{2}+d^{2}, 2|b, 2| d,(p / q)=+1$, and define

$$
A=p q, \quad B=b\left(c^{2}-d^{2}\right)+2 a c d, \quad C=a\left(c^{2}-d^{2}\right)-2 b c d, \quad m=q .
$$

Then $2 \mid B, B \equiv 2 d(a c+b d) \bmod q\left(\right.$ since $\left.c^{2} \equiv-d^{2} \bmod q\right)$, the sign of $A$ does not matter (since $q \equiv 1 \bmod 4$ ), and so formula (1) yields

$$
\left(\frac{q}{p}\right)_{4}=\left(\frac{A+B \sqrt{p}}{q}\right)=\left(\frac{B}{q}\right)\left(\frac{p}{q}\right)_{4}
$$

and the well-known $\left(\frac{2 d}{q}\right)=+1$ implies Burde's law

$$
\left(\frac{p}{q}\right)_{4}\left(\frac{q}{p}\right)_{4}=\left(\frac{a c-b d}{q}\right)
$$

A rational reciprocity law equivalent to Burde's has already been found by T. Gosset [5], who showed that, for primes $p$ and $q$ as above,

$$
\left(\frac{q}{p}\right)_{4} \equiv\left(\frac{a / b-c / d}{a / b+c / d}\right)^{(q-1) / 4} \bmod q
$$

Multiplying the numerator and denominator of the term on the right side of (8) by $a / b+c / d$ and observing that $c^{2} / d^{2} \equiv-1 \bmod q$ yields

$$
\begin{aligned}
\left(\frac{q}{p}\right)_{4} & =\left(\frac{a^{2} / b^{2}+1}{q}\right)_{4}\left(\frac{a / b+c / d}{q}\right)=\left(\frac{p}{q}\right)_{4}\left(\frac{b}{q}\right)\left(\frac{a / b+c / d}{q}\right) \\
& =\left(\frac{p}{q}\right)_{4}\left(\frac{a+b c / d}{q}\right)=\left(\frac{p}{q}\right)_{4}\left(\frac{d}{q}\right)\left(\frac{a d+b c}{q}\right),
\end{aligned}
$$

which is Burde's reciprocity law since $\left(\frac{2 d}{q}\right)=+1$. 
A more explicit form of Burde's reciprocity law for composite values of $p$ and $q$ has been given by L. Rédei [8]; letting $n=p q=A^{2}+B^{2}$ in $[8, \S 5$, (17), (19)], we find $A=a c-b d, B=a d+b c$, and his reciprocity formula $[8,(23)]$ gives our formula $(7)$.

Yet another version of Burde's law is due to A. Fröhlich [4]; he showed

$$
\left(\frac{p}{q}\right)_{4}\left(\frac{q}{p}\right)_{4}=\left(\frac{a+b j}{q}\right)=\left(\frac{c+d i}{p}\right)
$$

where $i$ and $j$ denote rational numbers such that $i^{2} \equiv-1 \bmod p, j^{2} \equiv$ $-1 \bmod q$. Letting $i=b / a$ and $j=d / c$ and observing that $\left(\frac{a}{p}\right)=\left(\frac{c}{q}\right)=+1$ we find that (9) is equivalent to (7).

The reciprocity law of Lehmer $[6,7]$ is even older; it can be found in Dirichlet's paper [2] as Théorème I and II; Dirichlet's ideas are reproduced in the charming book of Venkov [10] and may be used to give proofs for other rational reciprocity laws using nothing beyond quadratic reciprocity.

\section{References}

[1] K. Burde, Ein rationales biquadratisches Reziprozitätsgesetz, J. Reine Angew. Math. 235 (1969), 175-184.

[2] G. Lejeune Dirichlet, Recherche sur les diviseurs premiers d'une classe de formules du quatrième degré, ibid. 3 (1828), 35-69; Werke I, 63-98.

[3] R. Evans, Residuacity of primes, Rocky Mountain J. Math. 19 (1989), 1069-1081.

[4] A. Fröhlich, The restricted biquadratic residue symbol, Proc. London Math. Soc. (3) 9 (1959), 189-207.

[5] T. Gosset, On the law of quartic reciprocity, Mess. Math. (2) 41 (1911), 65-90.

[6] E. Lehmer, Criteria for cubic and quartic residuacity, Mathematika 5 (1958), 2029.

[7] —, Rational reciprocity laws, Amer. Math. Monthly 85 (1978), 467-472.

[8] L. Rédei, Über die Grundeinheit und die durch 8 teilbaren Invarianten der absoluten Klassengruppe im quadratischen Zahlkörper, J. Reine Angew. Math. 171 (1934), 131-148.

[9] A. Scholz, Über die Lösbarkeit der Gleichung $t^{2}-D u^{2}=-4$, Math. Z. 39 (1934), 95-111.

[10] B. A. Venkov, Elementary Number Theory, Moscow, 1937 (in Russian); English transl.: H. Alderson (ed.), Wolters-Noordhoff, Groningen, 1970.

[11] K. S. Williams, K. Hardy and C. Friesen, On the evaluation of the Legendre symbol $\left(\frac{A+B \sqrt{m}}{p}\right)$, Acta Arith. 45 (1985), 255-272.

ERWIN-ROHDE-STR. 19

69120 HEIDELBERG

GERMANY 